\title{
Polymorphisms of xenobiotic-metabolizing and transporter genes and the risk of gastric and colorectal cancer in an admixed population of Brazil.
}

Marianne Rodrigues Fernandes

Universidade do Estado do Para

Amanda de Nazaré Cohen Lima de Castroq

Universidade do Estado do Para

Darlen Cardoso de Carvalho

Universidade Federal do Para

Tatiane Piedade de Souza

Universidade Federal do Para

Juliana Carla Gomes Rodrigues

Universidade Federal do Para

Roberta Borges Andrade

Universidade Federal do Para

Antonio André Conde Modesto

Hospital Universitario Joao de Barros Barreto

Sidney Emanuel Batista dos Santos

Universidade Federal do Para

Paulo Pimentel de Assumpção

Hospital Universitario Joao de Barros Barreto

Ney Pereira Carneiro dos Santos ( $\sim$ npcsantos.ufpa@gmail.com )

UNIVERSIDADE FEDERAL DO PARÁ https://orcid.org/0000-0003-3548-292X

Research article

Keywords: Metabolism, xenobiotic, polymorphism, susceptibility, admixed

Posted Date: September 9th, 2019

DOI: https://doi.org/10.21203/rs.2.14223/v1

License: (c) (1) This work is licensed under a Creative Commons Attribution 4.0 International License.

Read Full License 
Page 2/15 


\section{Abstract}

Introduction

Colorectal (CRC) and Gastric (GC) cancers are responsible for considerable morbidity and mortality worldwide. In the North region of Brazil, these neoplasms are among the three most incident and aggressive types of cancer, constituting a severe problem of public health. Single Nucleotide Polymorphisms (SNPs) of xenobiotic metabolism and transporter genes may play a role in individual response to exposure to some of the compounds implicated in the cancer susceptibility. However, few studies have demonstrated the role of polymorphisms of xenobiotic metabolism and transporter genes in the susceptibility to CRC or GC in admixed populations. In this context, the study of variation in the activation and detoxification processes of xenobiotics may help to clarify the development of either GC or CRC in substructured populations, providing new insights about predictive diagnostic criteria in oncological investigations.

Methods

We performed an association study using 31 SNPs in 15 xenobiotic metabolism and transporter genes. The study was carried out in $121 \mathrm{CRC}$ and $95 \mathrm{GC}$ cases and 140 control individuals from Belém, a city which comprises a population with high levels of miscegenation, located in the Brazilian Amazon. Samples were genotyped using the QuantStudio ${ }^{\text {TM }} 12 \mathrm{~K}$ Flex Real-Time PCR System. Due to the high level of genetic admixture in the studied population, we applied a panel of 61 Ancestry Informative Marker standardized by our research group in an earlier study. Statistical analyses were performed in SPSS v.20.0 and Structure v.2.3.4

Results

The results revealed a significant association between the increased CRC or GC risk and polymorphisms of $A B C G 2$ (rs2231142) and DPYD genes (rs17116806, rs1801159).

Conclusions

Our data suggest that polymorphisms in xenobiotic-metabolizing and transporter genes may be relevant to the susceptibility to both CRC and GC.

\section{Introduction}

Gastric and colorectal cancers are the main causes of cancer deaths worldwide [1] [2]. In northern Brazil, the incidence of these types of neoplasm is relatively high in comparison with the average rate observed in other regions of the country [2]. The carcinogenesis of colorectal (CRC) and gastric (GC) cancers is still unclear. A number of studies have indicated that the exposure to several environmental or lifestyle risk factors have been identified for CRC and GC, such as obesity, sedentary behavior, high intake of hypercaloric foods, diets rich in fat and poor in fiber, tobacco smoking and alcohol consumption [3][4][5] 
[6]. In addition, the genetic makeup is hypothesized to play an important role in the sporadic form of these neoplasms. Single nucleotide polymorphisms (SNPs) are one of the most common types of genetic variations in the human genome, and from a clinical perspective, are potential diagnostic and therapeutic biomarkers in many cancer types. SNPs in xenobiotic metabolizing and transporter genes are known to modify the activity of their encoded enzymes, resulting in an increase or decrease predisposition (depending on the pathway of each enzyme) for the development of gastric or colorectal neoplasms [6][7] [8][9]. However, few studies have demonstrated the role of polymorphisms in xenobiotic metabolism and transporter genes in the susceptibility to CRC or GC in admixed populations, such as the Brazilian one. This factor is highly relevant because if subjects of a genetic association study are sampled from two or more subpopulations for which the frequencies of alleles and traits differ; spurious associations may arise due to the population substructure [10].

Given this, the goal of this work was to investigate the role of 31 polymorphisms in 15 xenobiotic metabolizing and transporter genes and the influence of genetic ancestry in CRC and GC susceptibility in an population from the Brazilian Amazon region with a high degree of interethnic admixture.

\section{Patients And Methods}

Study participants

In this study, we included three groups: (1) 95 individuals with GC diagnosis; (2) 121 individuals with CRC diagnosis; and (3) 140 cancer-free individuals. The cancer-free individuals did not have personal or familial histories of any type of cancer and they did not show any symptoms or signs of cancer. All individuals resided in Belém, which is a city located in the northern region of Brazil. All patients were treated at two local hospitals: Ophir Loyola Hospital and University Hospital João de Barros Barreto. All selected participants were from the same geographical area and groups of similar socioeconomic status.

Ethics, consent and permissions

All participants gave written informed consent to participate and publish this investigation. The protocol used in the study was approved by the Ethics Committee of the University Hospital João de Barros Barreto (protocol number 231.244/2013) and Ophir Loyola Hospital (298.994/2013).

DNA extraction and quantification

Peripheral blood samples were collected from all individuals of the study and the DNA extraction was performed with commercial kit Biopur KitPlus Mini Spin Extract-250 (Biopur, Brazil) according to the manufacturer's instructions. DNA quantification was performed with NanoDrop 1000 spectrophotometer (Thermo Scientific, Wilmington, DE, United States).

Analysis of the polymorphisms 
Samples were genotyped using QuantStudio ${ }^{\text {TM }} 12 \mathrm{~K}$ Flex Real-Time PCR System by TaqMan Open Array Genotyping (Applied Biosystems, Life Technologies, Carlsbad, USA), according to the protocol published by Applied Biosystems.

Analysis of genetic ancestry

To avoid misinterpretations caused by a high level of genetic admixture in the studied population, we applied a panel of 61 ancestry-informative markers as described previously [11]. The ancestral populations included representatives of three major ethnic groups, including Amerindian from the Brazilian Amazon, African and European populations. More details on these populations can be found in [12]. The genomic ancestry was performed in Structure v.2.3.4 software.

Statistical analysis

The association analyses were performed in SPSS v.20.0 (IBM Corp., Armonk, NY, United States) using Student's t-test, Pearson's $\chi 2$ test, Mann-Whitney test and logistic regression. The genotype distribution was assessed as established by Hardy-Weinberg equilibrium (HWE). A $p$-value $\leq 0.05$ was considered statistically significant.

\section{Results}

Demographic characteristics

A total of 356 individuals were analyzed in the present study. Demographic data about case and control groups are presented in Table 1 and Table 2, for GC and CRC, respectively. Regarding sex, the CG case group was predominantly composed of men. Differently, the CRC case group had a predominance of women. Significant differences were found between the two groups (GC and CRC) related to age and sex, therefore, these variables were controlled for in the logistic regression. Genomic ancestry analysis for the CG group showed $45 \%$ of European, $22 \%$ African and $33 \%$ Amerindian ancestries.

The same analysis for CRC patients demonstrated 50\% European, 30\% Amerindian, and 20\% African ancestries. No significant association was found in the genomic ancestry analysis for GC or CRC.

Quality control and Genotyping

A total of 31 polymorphisms were investigated in this study (Table S1). Six variants were excluded from our analysis: nine polymorphisms were out of Hardy-Weinberg Equilibrium and three marker was above the limit of $15 \%$ of missing data in Minor Allele Frequency (MAF) analysis. The remaining 19 SNPs were analyzed. Table S2 shows the HWE and MAF data of the 31 polymorphisms and their study status (included or excluded).

Analysis of associations with susceptibility to GC and CRC 
Significancy associations were found with the investigated polymorphisms in relation to an increased GC risk (Table 3). The results indicated that rs2231142 variant of the $A B C G 2$ transporter gene increases in about 3 times the risk for developing GC $(\mathrm{p}=0.013 ; \mathrm{OR}=3.01,95 \% \mathrm{Cl}=1.26-7.13)$. Regarding the $D P Y D$ polymorphisms, the rs1801159 variant demonstrated to increase the risk to develop GC $(p=0.03, O R=$ $2.35,95 \% \mathrm{Cl}=1.14-5.05)$. Similarly, the rs 17116806 polymorphism demonstrated to confer greater susceptibility for the development of this cancer type $(p=<0.0001 ; \mathrm{OR}=23.25 ; 95 \% \mathrm{Cl}=66.6-8.33)$. Individuals homozygous for AA mutant genotype in the rs17116806 of the DPYD gene have an approximately 23-fold increased risk for GC development over those with other genotypes. Interestingly, the same polymorphism has been shown the same effect to colorectal carcinogenesis $(p=<0.0001 ; O R=$ $1.31 ; 95 \% \mathrm{Cl}=30.3-5.5)$. The data of the other polymorphisms investigated that did not present statistical significance are shown in the supplementary material (Table S3 and Table S4).

\section{Discussion}

Colorectal and gastric cancers are among the leading causes of death worldwide [1]. In Brazil, estimates for 2018-2019 from the National Cancer Institute (INCA) indicate more than 417000 new cases of these malignant neoplasms [2]. In the northern region of the country, where our target population resides, GC is the second most prevalent type of neoplasia in men and the fourth in women, whereas the CRC is the fourth most incident in men and the third in women [2].

Although the causes of cancer have not yet been completely elucidated, studies have shown that a large group of mutagen-carcinogenic agents require metabolic activation to allow them to bind to DNA, RNA and proteins; therefore, several environmental components are strong risk factors for GC and CRC development [13][14]. Tomasetti (2017) demonstrated that the percentage of influence of external factors on GC and CRC susceptibility is $55 \%$ and $26 \%$, respectively [15], thus, association studies in genetic pathways related to the metabolism and transport of environmental risk factors have aided to better understand the carcinogenesis process in several organs [16][17][18]. Most genetic association studies on cancer are investigations about tumor suppressors or oncogenes. Our analysis, however, proposes the study of xenobiotic-metabolizing and transporter genes, which can also modulate the susceptibility to different types of cancer. Additionally, few of these studies have been performed in admixed populations such as the Brazilian one. The Brazilian population is composed, mainly, by the admixture of Amerindian, European, and African ancestral populations [11][12]. Case-control studies in such admixed populations may be influenced by the variation of allelic frequencies of markers found in each different ethnic groups, which may create biases in the outcomes, especially in investigations of susceptibility to complex diseases, such as cancer [19][20]. In this case, estimates of ethnic admixture must be taken into consideration. Our investigation of genomic ancestry analysis was based on the set of 61 AIMs used in previous genetic studies of complex human diseases[11]. Our research group have performed some studies that demonstrated the influence of population substructure present in the northern region of Brazil with several types of cancer, for example childhood B-cell Leukemia [20][21] and breast and gastric cancer [7]. In the present study, however, no significant difference was found in the ethnic profiles between the case and control groups. 
In this study, the polymorphisms shown to be associated with colorectal or gastric cancers are related to xenobiotic metabolism. A major group of mutagenic-carcinogenic agents requires metabolic activation to enable them to bind to DNA, RNA, and proteins. Therefore, genetic polymorphisms in these xenobioticmetabolizing and transporter genes may account for the individual variation observed in the individual response to exposure [18][19][20].

In our analysis, the rs2231142 variant of the $A B C G 2$ gene increased approximately 3 times the risk forGC development. The $A B C G 2$ gene encodes the Human Breast Cancer resistance protein (BCRP)/ATP-binding cassette subfamily $\mathrm{G}$ member 2 ( $A B C G 2)$,, which is an ATP-binding cassette (ABC) transporter responsible for the active transport of several compounds through extra and intracellular membranes [22]. This protein expression occurs predominantly in the liver and in the apical membrane of the intestinal epithelium, playing an important role in intestinal absorption and mediation of hepatobiliary excretion of its substrates (such as potentially carcinogenic xenobiotics and anticancer drugs) [23]. The BRCP is known as a molecular cause of multidrug resistance (MDR) in several cancer cells, but recently some research has focused on understanding its role as a susceptibility biomarker of human carcinoma cells [24][25]. Gupta et al. found a decrease in mRNA expression of ABCG2 in colorectal and cervical cancer, suggesting a role of this gene in tumorigenesis through the accumulation of genotoxins and the excess of nitric oxide production, which the author suggests being a common phenomenon in other tissues where this gene is hypo-expressed [26]. Supporting this finding, the study by Liu et al. (2010) demonstrated a differential expression of the BCRP at each carcinogenesis stage [27]. For the promotion of carcinogenesis, the expression of BCRP would be decreased to allow the accumulation of genotoxins and nitric oxide, but in the more advanced stages, BRCP can be positively expressed to efficiently transport chemotherapeutic drugs out of the cancerous cells, protecting them. Therefore, the assessment of differential expressions of BRCP may lead the scientific community to create a new method to evaluate progression, metastasis and to predict therapeutic response of colorectal cancer. The results also corroborate with the findings of Wang and colleagues, who, through an in vitro experiment, demonstrated that the $A B C G 2$ gene shows dysregulated expression in GC tissues and cells [28]. In this study, the high expression of $A B C G 2 / B R C P$ was correlated with advanced stages and poor prognosis of $\mathrm{GC}$. Deregulated expression of the $A B C G 2$ gene has further been pointed as a promoter factor to $\mathrm{GC}$ that affects cell proliferation and induces resistance to cellular apoptosis. These results corroborate with our analyses, confirming the role of SNPs of the $A B C G 2$ gene in the initiation and promotion of GC.

Polymorphisms in the DPYD gene have also been shown to play a role in gastric or colorectal carcinogenesis. Our data demonstrated that the rs1801159 and rs17116806 polymorphisms are associated with a higher risk of GC susceptibility. Additionally, the rs 17116806 has been also associated to an increased susceptibility to colorectal carcinogenesis, demonstrating that the same polymorphism can act on tumorigenesis in different tissues. International regulatory agencies, such as the US Food and Drug Administration (FDA) and the European Medicines Agency (EMA), strongly recommend the monitoring of polymorphisms on the DPYD gene for evaluation of therapeutic response in fluoropyrimidine-based treatment [29][30]. Nevertheless, few investigations have studied genetic variations in the DPYD gene regarding cancer susceptibility, thus, the variety of clinical manifestations 
resulting by mutations in DPYD is still not well understood [31]. To date, there are no genetic association studies with the rs1801159 and rs17116806 polymorphisms and the susceptibility to the neoplasms investigated hereby. Therefore, this study is the first to investigate the correlation between DPYD polymorphisms and the susceptibility to GC and CRC in the Brazilian Amazon population.

Previous studies have also shown that modifications of the pyrimidines homeostasis and the products of their degradation can result in a number of phenotypic manifestations, including neurological disturbances [32] and gastrointestinal disorders [31]. Tanaka et al. analyzed the rs1801265 variant and the risk to develop six different neoplasms: esophagus, gastric, colon, lung, breast, and lymphomas, suggesting an influence of this variant on the development of these types of cancer [33]. Matáková et al. (2017) demonstrated a significant association with the rs 1801160 SNP of the DPYD and an increased risk for the CRC development $(p=0.003, \mathrm{OR}=3.264,95 \% \mathrm{Cl}=1.425-7.475)$ [34]. Edward et al. (2016) showed a comprehensive view of how the polymorphisms in the DPYD gene deregulate the pyrimidine and nucleic acid synthesis, consequently, promoting malignant progression of melanoma [35]. A multicenter study concluded that variations in genes involved in the metabolism of pyrimidines, particularly the $D P Y D$, may also influence the susceptibility to ovarian carcinoma [36].

From the findings to date, we can infer that relevant clinical interventions is possible by enhancing the knowledge of the basic set of mutations that can lead to gastric and colorectal carcinogenesis. This outcomes creates a new expectation regarding the progress of studies related to the predictive diagnosis of cancer.

\section{Conclusion}

In conclusion, our data demonstrate that SNPs in the $A B C G 2$ and DPYD play an important role in gastric and colorectal susceptibility in a highly substructured population from the Brazilian Amazon. The obtained results may help to clarify the genetic factors underlying the GC or CRC development in the studied population.

\section{List Of Abbreviations}

- CRC: Colorectal Cancer

- GC: Gastric Cancer

- SNP: Single nucleotide polymorphisms

\section{Declarations}

Acknowledgments

We thank Dr. Artur Luiz da Costa Silva for his collaboration in the use of the equipment structure of the Parque de Ciência e Tecnologia (PCT-Guamá/ UFPA); Msc. Daniele Feio da Costa and Williams Fernandes 
Barra in the assistance of the sample collection and clinical data from patients and Hospital Universitário João de Barros Barreto (HUJBB/ UFPA) and Hospital Ophir Loyola (HOL/ PA).

Funding

This study was financed by the Coordenação de Aperfeiçoamento de Pessoal de Nível Superior - Brasil (CAPES) - Finance Code 001, Programa de auxílio à pesquisa - Projetos temáticos from Fundação Amazônia de Amparo a Estudos e Pesquisas. The Conselho Nacional de Desenvolvimento Científico e Tecnológico (FAPESPA / CNPq): Saúde, No. 006/2014 financed the purchase of consumer material used in the methodology and scholarship studies and Pró-Reitoria de Pesquisa e Pós-Graduação da Universidade Federal do Pará (PROPESP / UFPA) funded the publication's fee.

Authors' contributions

ANCLC, MRF and NPCS designated the study. ANCLC, MRF, JCGR and AACM conducted the molecular genetic study. ANCLC, MRF, DCC and RBA participated in the statistical analyses. ANCLC and MRF wrote the manuscript. DCC and TPS reviewed the manuscript and citations. PPA, SEBS and NPCS were project's coordinators. All the authors have read and approved the final manuscript.

Ethics approval and consent to participate

All participants gave written informed consent. The protocol used in the study was approved by the Ethics Committee of the University Hospital João de Barros Barreto (protocol number 231.244/2013) and Ophir Loyola Hospital (298.994/2013).

Consent for publication

All participants consented to the study consented for publication.

Competing interests

All authors have no competing interests with regards to the study. No potential conflicts of interest.

\section{References}

1. Steward, B. and Wild, C. P. (eds.). International Agency for research on cancer, WHO. (2014) World Cancer Report 2014 [Online]. Available from: http://publichealthwell.ie/node/725845 [Acessed: 11th July 2019].

2. Instituto Nacional do Cancer. (2017). Estimativa 2018- Incidência de câncer no Brasil/Instituto Nacional de Câncer. Available from: http://www.inca.gov.br/estimativa/2018.

3. Ma G, Liu H, Du M, Zhang G, Lin Y, Ge Y, et al. A genetic variation in the CpG island of pseudogene GBAP1 promoter is associated with gastric cancer susceptibility. Cancer. 2019; doi:10.1002/cncr.32081. 
4. Barontini J, Antinucci M, Tofanelli S, Cammalleri M, Monte M, Gemignani F, et al. Association between polymorphisms of TAS2R16 and susceptibility to colorectal cancer. BMC Gastroenterol. 2017; 17: 104.

5. Sari F, Yanar H, Ozhan G. Investigation of the functional single-nucleotide Liu J, Zheng B, Li Y, Yuan Y, Xing C. Genetic Polymorphisms of DNA Repair Pathways in Sporadic Colorectal Carcinogenesis. J Cancer. 2019 Feb 23;10(6):1417-1433. doi: 10.7150/jca.28406.

6. Baroudi O, Benammar-Elgaaied A. Involvement of genetic factors and lifestyle on the occurrence of colorectal and gastric cancer. Crit Rev Oncol Hematol. 2016 Nov;107:72-81. doi: 10.1016/j.critrevonc.2016.08.014.

7. Fernandes, M. R., De Carvalho, D.C., Dos Santos, Â. K. C. R., Dos Santos, S. E. B., De Assumpção, P. P., Burbano, R. M. R., \& Dos Santos, N. P. C. (2013). Association of slow acetylation profile of NAT2 with breast and gastric cancer risk in Brazil. Anticancer research, 33(9), 3683-3689.

8. Tang C, Yu S, Jiang H, Li W, Xu X, Cheng X, Peng K, Chen E, Cui Y, Liu T. A Meta-Analysis: Methylenetetrahydrofolate Reductase C677T Polymorphism in Gastric Cancer Patients Treated with 5-Fu Based Chemotherapy Predicts Serious Hematologic Toxicity but Not Prognosis. J Cancer. 2018 Feb 28;9(6):1057-1066. doi: 10.7150/jca.23391.

9. Cuyle PJ, Prenen H. Current and future biomarkers in the treatment of colorectal cancer. Acta Clin Belg. 2017 Apr; 72(2):103-115. doi: 10.1080/17843286.2016.1262996.

10. Santos NP, Ribeiro-Rodrigues EM, Ribeiro-Dos-Santos AK, Pereira R, Gusmão L, Amorim, et al. Assessing individual interethnic admixture and population substructure using a 48-insertiondeletion (INSEL) ancestry-informative marker (AIM) panel. Human mutation. 2010. 31(2), 184-190. doi: 10.1002/humu.21159.

11. Ramos, B. R. A., Mendes, N. D., Tanikawa, A. A., Amador, M. A. T., Santos, N. P. C., Santos, S. E. B., et al. Ancestry informative markers and selected single nucleotide polymorphisms in immunoregulatory genes on preterm labor and preterm premature rupture of membranes: a case control study. BMC Pregnancy and Childbirth. 2016 Feb 5;16:30. doi: 10.1186/s12884-016-0823-1.

12. Amador MAT, Cavalcante GC, Santos NPC, Gusmão L, Guerreiro JF, Ribeiro-dos-Santos Â, et al. Distribution of allelic and genotypic frequencies of IL1A, IL4, NFKB1 and PAR1 variants in Native American, African, European and Brazilian populations. BMC Res Notes. BioMed Central; 2016;9:101. doi: 10.1186/s13104-016-1906-9.

13. Shi-Heng Zhang, Lin-Ang Wang, Zheng Li, Yu Peng, Yan-Ping Cun, Nan Dai, et al. APE1 polymorphisms are associated with colorectal cancer susceptibility in Chinese Hans. World J Gastroenterol. 2014; 20(26): 8700-8708. doi: 10.3748/wjg.v20.i26.8700.

14. Xia LZ, Liu Y, Xu XZ, Jiang PC, Ma G, Bu XF, et al. Methylenetetrahydrofolate reductase C677T and A1298C polymorphisms and gastric cancer susceptibility. World J Gastroenterol. 2014;20(32): 11429-11438. doi: 10.3748/wjg.v20.i32.11429.

15. Tomasetti C, Li L,Vogelstein B. Stem cell divisions, somatic mutations, cancer etiology, and cancer prevention. Science. 2017;355(6331):1330-1334. doi: 10.1126/science.aaf9011. 
16. Schumacher FR, Al Olama AA, Berndt SI, Benlloch S, Ahmed M, Saunders EJ, et al. Association analyses of more than 140,000 men identify 63 new prostate cancer susceptibility loci. Nat Genet. 2018;50(7):928-936. doi: 10.1038/s41588-018-0142-8.

17. Cavalcante GC, Amador MA, Ribeiro Dos Santos AM, Carvalho DC, Andrade RB, Pereira EE, et al. Analysis of 12 variants in the development of gastric and colorectal cancers. World J Gastroenterol. 2017;23(48):8533-8543. doi: 10.3748/wjg.v23.i48.8533.

18. Ji X, Bossé Y, Landi MT, Gui J, Xiao X, Qian D, et al. Identification of susceptibility pathways for the role of chromosome 15q25.1 in modifying lung cancer risk. Nat Commun. 2018;9(1):3221. doi: 10.1038/s41467-018-05074-y.

19. Wang H, Schmit SL, Haiman CA, Keku TO, Kato I, Palmer JR, Hispanic Colorectal Cancer Study, Le Marchand L. Novel colon cancer susceptibility variants identified from a genome-wide association study in African Americans. Int J Cancer. 2017 Jun 15;140(12):2728-2733. doi: 10.1002/ijc.30687.

20. Carvalho, D.C., Wanderley, A. V., Amador, M. A., Fernandes, M. R., Cavalcante, G. C., Pantoja, K. B., \& Santos, S. (2015). Amerindian genetic ancestry and INDEL polymorphisms associated with susceptibility of childhood B-cell Leukemia in an admixed population from the Brazilian Amazon. Leukemia research, 39(11), 1239-1245. doi: 10.1016/j.leukres.2015.08.008.

21. Carvalho DC, Wanderley AV, Dos Santos AMR, Fernandes MR, Cohen Lima de Castro AN, Leitão LPC, de Carvalho JAN Junior, de Souza TP, Khayat AS, Dos Santos SEB, de Assumpção PP, Dos Santos NPC. Pharmacogenomics and variations in the risk of toxicity during the consolidation/maintenance phases of the treatment of pediatric B-cell leukemia patients from an admixed population in the Brazilian Amazon. Leuk Res. 2018 Nov;74:10-13.

22. Velamakanni S, Wei SL, Janvilisri T, van Veen HW. 6-8ABCG transporters: structure, substrate specificities and physiological roles: a brief overview. Bioenerg Biomembr. 2007;39(5-6):465-71. doi: 10.1007/s10863-007-9122-x.

23. Sari FM, Yanar HT, Ozhan G. Investigation of the functional single-nucleotide polymorphisms in the BCRP transporter and susceptibility to colorectal câncer. Biomed Rep. 2015;3(1): 105-109. doi: 10.3892/br.2014.383.

24. Wang J, Yunyun Z, Wang L, Chen X, Zhu Z. ABCG2 confers promotion in gastric cancer through modulating downstream CRKL in vitro combining with biostatistics mining. Oncotarget. 2017;8(3): 5256-5267. doi: 10.18632/oncotarget.14128.

25. Kondo C, Suzuki H, Itoda M, Ozawa S, Sawada J, Kobayashi D, et al. Functional analysis of SNPs variants of BCRP/ABCG2. Pharm Res. 2004;21(10):1895-903.

26. Gupta N, Martin PM, Miyauchi S, Ananth S, Herdman AV, Martindale RG, et al. Down-regulation of BCRP/ABCG2 in colorectal and cervical cancer. Biochem Biophys Res Commun. 2006;343(2):571-7. doi: 10.1016/j.bbrc.2006.02.172.

27. Liu HG, Pan YF, You J, Wang OC, Huang KT, Zhang XH. Expression of ABCG2 and its significance in colorectal cancer. Asian Pac J Cancer Prev. 2010;11(4):845-8. 
28. Wang J, Yunyun Z, Wang L, Chen X, Zhu Z. ABCG2 confers promotion in gastric cancer through modulating downstream CRKL in vitro combining with biostatistics mining. Oncotarget. 2017;8(3):5256-5267. doi: 10.18632/oncotarget.14128.

29. Zhang H, Li YM, Zhang H, Jin X. DPYD*5 gene mutation contributes to the reduced DPYD enzyme activity and chemotherapeutic toxicity of 5-FU: results from genotyping study on 75 gastric carcinoma and colon carcinoma patients. Med Oncol. 2007;24(2):251-8.

30. Henricks LM, Opdam FL, Beijnen JH, Cats A, Schellens JHM. DPYD genotype-guided dose individualization to improve patient safety of fluoropyrimidine therapy: call for a drug label update. Ann Oncol. 2017;28(12):2915-2922. doi: 10.1093/annonc/mdx411.

31. Jurecka A. Inborn errors of purine and pyrimidine metabolism. J Inherit Metab Dis. 2009;32(2):24763. doi: 10.1007/s10545-009-1094-z.

32. van Kuilenburg AB, Dobritzsch D, Meijer J, Meinsma R, Benoist JF, Assmann B, et al. Dihydropyrimidinase deficiency: Phenotype, genotype and structural consequencesin 17 patients. Biochim Biophys Acta. 2010 Jul-Aug;1802(7-8):639-48. doi:10.1016/j.bbadis.2010.03.013.

33. Tanaka D, Hishida A, Matsuo K, Iwata H, Shinoda M, Yamamura Y, et al. Polymorphism of dihydropyrimidine dehydrogenase (DPYD) Cys29Arg and risk of six malignancies in Japanese. Nagoya J Med Sci. 2005;67(3-4):117-24.

34. Matáková T, Halašová E, Škovierová H, Dzian A, Dobrota D, Škereňová M. DPYD genotype and haplotype analysis and colorectal cancer susceptibility in a case-control study from Slovakia. Gen Physiol Biophys. 2017;36(5):557-563. doi: 10.4149/gpb_2017046.

35. Edwards L, Gupta R, Filipp FV. Hypermutation of DPYD Deregulates Pyrimidine Metabolism and Promotes Malignant Progression. Mol Cancer Res. 2016;14(2):196-206. doi: 10.1158/15417786.MCR-15-0403.

36. Kelemen LE, Terry KL, Goodman MT, Webb PM, Bandera EV, McGuire V, et al. Consortium analysis of gene and gene-folate interactions in purine and pyrimidine metabolism pathways with ovarian carcinoma risk. Mol Nutr Food Res. 2014;58(10):2023-35. doi:10.1002/mnfr.201400068.

\section{Tables}

Table 1. Demographic variables for patients with gastric cancer and control group.

\begin{tabular}{lccc}
\hline Variable & Case & Control & $p$-value \\
\hline No. & 95 & 140 & \\
Age, years $^{\mathrm{a}}$ & $55.59 \pm 9.94$ & $69,44 \pm 6,89$ & $0.000^{\mathrm{b}}$ \\
\hline Gender (Female/Male) $^{\text {G }}$ & $36 / 59$ & $101 / 39$ & $<0.001$ \\
\hline Genetic Ancestry $^{\mathrm{a}}$ & & & \\
\hline European & $0.45 \pm 0.16$ & $0.45 \pm 0.17$ & $0.87^{\mathrm{b}}$ \\
\hline African & $0.22 \pm 0.12$ & $0.23 \pm 0.14$ & $0.68^{\mathrm{b}}$ \\
\hline Amerindian & $0.33 \pm 0,13$ & $0.32 \pm 0.15$ & $0.21^{\mathrm{b}}$ \\
\hline & Page $12 / 15$ & &
\end{tabular}




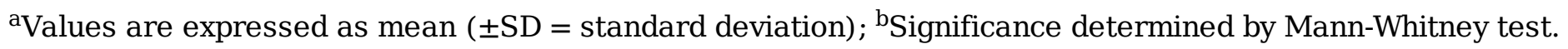

Table 2. Demographic variables for patients with colorectal cancer and control group.

\begin{tabular}{lccc}
\hline Variable & Case & Control & $p$-value \\
\hline No. & 121 & 140 & \\
Age, years $^{\mathrm{a}}$ & $54.05 \pm 12.06$ & $69.44 \pm 6.89$ & $0.000^{\mathrm{b}}$ \\
\hline Gender (Female/Male) $^{\mathrm{b}}$ & $67 / 54$ & $101 / 39$ & 0.006 \\
\hline Genetic Ancestry $^{\mathrm{a}}$ & & & \\
\hline European & $0.50 \pm 0.14$ & $0.45 \pm 0.17$ & $0.015^{\mathrm{b}}$ \\
\hline African & $0.20 \pm 0.10$ & $0.23 \pm 0.14$ & $0.11^{\mathrm{b}}$ \\
\hline Amerindian & $0.30 \pm 0,12$ & $0.32 \pm 0.15$ & $0.77^{\mathrm{b}}$ \\
\hline
\end{tabular}

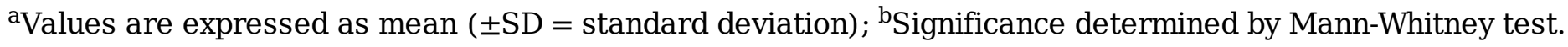

Table 3. Genotype distribution of the investigated polymorphisms in patients with gastric or colorectal cancer in comparison with the control group. 


\begin{tabular}{|c|c|c|c|c|c|}
\hline Cancer type & Genotype & No. (\%) Case & No. (\%) Control & $p$-value ${ }^{\mathrm{a}}$ & $\begin{array}{l}\text { OR } \\
(95 \% \text { IC })^{a}\end{array}$ \\
\hline \multirow[t]{6}{*}{$G C$} & ABCG2 (rs2231142) & 93 & 122 & 0.013 & \\
\hline & GG & 65 (69.9\%) & $68(55.7 \%)$ & & GG vs. others: \\
\hline & GT & 25 (26.9\%) & $51(41.8 \%)$ & & $3.01(1.26-7.13)$ \\
\hline & $\mathrm{TT}$ & $3(3.2 \%)$ & $3(2.5 \%)$ & & \\
\hline & Allele $G$ & 0.8 & 0.77 & & \\
\hline & Allele $T$ & 0.2 & 0.23 & & \\
\hline \multirow[t]{6}{*}{$G C$} & $D P Y D \quad$ (rs1801159) & 94 & 134 & 0.03 & \\
\hline & $\mathrm{TT}$ & $55(58.5 \%)$ & 49 (36.6\%) & & TT vs. others: \\
\hline & $\mathrm{TC}$ & $35(37.2 \%)$ & 76 (56.7\%) & & $2.35(1.14-5.05)$ \\
\hline & $\mathrm{CC}$ & $4(4.3 \%)$ & $9(6.7 \%)$ & & \\
\hline & Allele $T$ & 0.8 & 0.6 & & \\
\hline & Allele $C$ & 0.2 & 0.4 & & \\
\hline \multirow[t]{6}{*}{$G C$} & $D P Y D($ rs 17116806) & 92 & 121 & & \\
\hline & $\mathrm{CC}$ & 35 (38\%) & 95 (78.5\%) & $<0.0001$ & AA vs. others: \\
\hline & CA & $41(44.6 \%)$ & $26(21.5 \%)$ & & $23.25(66.6-8.33)$ \\
\hline & AA & $16(17.4 \%)$ & $0(0.0 \%)$ & & \\
\hline & Allele $C$ & 0.6 & 0.9 & & \\
\hline & Allele $A$ & 0.4 & 0.1 & & \\
\hline \multirow[t]{6}{*}{$C R C$} & $D P Y D($ rs17116806) & 112 & 121 & & \\
\hline & $\mathrm{CC}$ & 29 (25.9\%) & 95 (78.5\%) & $<0.0001$ & AA vs. others: \\
\hline & CA & 57 (50.9\%) & 26 (21.5\%) & & $1.31(30.3-5.5)$ \\
\hline & AA & $26(23.2 \%)$ & $0(0.0 \%)$ & & \\
\hline & Allele $C$ & 0.5 & 0.9 & & \\
\hline & Allele $A$ & 0.5 & 0.1 & & \\
\hline
\end{tabular}

${ }^{a}$ Logistic regression adjusted for confounders: age and sex.

\section{Supplementary Files}

This is a list of supplementary files associated with this preprint. Click to download.

- TableSup3gastriccancerallelefrequency.docx

- TableSup4colorectalcancerallelefrequency.docx 
- TableSup1Characteristicsofthe31SNPs.docx

- TableSup2HWEeMAF.docx 